\title{
Ist die Statistik der santésuisse «psychiatrietauglich»?
}

\author{
H. P. Wieland
}

\section{Zusammenfassung}

Die Rechnungsstellerstatistik der Krankenversicherer hat in bezug auf die Wirtschaftlichkeitskontrolle psychiatrisch-psychotherapeutischer Behandlungen heftige Kontroversen ausgelöst. Die statistischen Kriterien sind fragwürdig, weil sie der Spezifität des Faches Psychiatrie-Psychotherapie nicht gerecht werden. Damit wird auch die wissenschaftliche Grundlage der Statistik zweifelhaft. Psychiaterinnen und Psychiater wehren sich nun dagegen, dass ihr Behandlungsspielraum durch eine untaugliche Indexabhängigkeit zum Schaden ihrer Patientinnen und Patienten eingeengt wird.

\section{Psychiatrie-Psychotherapie im Visier der santésuisse}

Die Diskussion über die Rechnungsstellerstatistik der Krankenversicherer ist seit Herbst 2002 wieder aktuell geworden, nachdem die santésuisse Wirtschaftlichkeitskontrollverfahren gegen einige psychiatrisch-psychotherapeutische Praxen eingeleitet hat. Das Problem ist jedoch schon länger virulent und es drängen sich deshalb einige grundsätzliche Fragen auf.

\section{Fragwürdige statistische Kriterien}

Die santésuisse zählt in einem Artikel in der Schweizerischen Ärztezeitung ihre Kriterien auf, nach welchen sie die Kosten ärztlicher Behandlungen mittels der «Rechnungsstellerstatistik der Krankenversicherer» kontrolliert [1]. So werden in der Statistik neben den Arztrechnungen auch Daten über die «Ausbildung und Spezialität», die «örtlichen Verhältnisse», die «Zusammensetzung der Patientinnen und Patienten (insbesondere Durchschnittsalter)» und die «weiteren als bedeutsam taxierten Faktoren, soweit solche bekannt sind», erfasst. Die Kriterien, auf welche sich die statistischen Untersuchungen der santésuisse stützen sollen, genügen jedoch einer Abbildung der komplexen Verhältnisse einer psychiatrischpsychotherapeutischen Praxis auf keinen Fall, weshalb sich folgende Fragen stellen.

\section{Mangelnde Differenziertheit in bezug auf die Psychiatrie-Psychotherapie}

Welches sind diese «als bedeutsam taxierten Faktoren»? Was heisst «soweit solche bekannt sind»? Wem sind sie bekannt bzw. nicht bekannt?

Sind den Statistikerinnen und Statistikern der santésuisse die speziellen Verhältnisse psychiatrisch-psychotherapeutischer Behandlungen bekannt? Ist ihnen bekannt, dass nicht alleine die «Ausbildung und Spezialität», sondern dass die individuellen Patienten- und Therapeutenpersönlichkeiten - welche letztere im fachlichen Weiterbildungscurriculum besondere Beachtung finden! - bedeutsame Faktoren darstellen? Ist ihnen bekannt, dass das «Durchschnittsalter» der Patientinnen und Patienten in der Psychiatrie-Psychotherapie eine geringere Rolle spielt als z. B. die Persönlichkeitsstruktur und Psychopathologie?

Ist den Statistikerinnen und Statistikern bekannt, dass solche differenzierte Faktoren selbst für mehr oder weniger identisch diagnostizierte Fälle nicht einheitlich gleich beurteilt werden können, da jede einzelne Patientin und jeder einzelne Patient selbst wiederum von weiteren individuellen und hochkomplexen Elementen im Verhalten geprägt ist? Ist ihnen bekannt, dass unter diesen Voraussetzungen jede Psychiaterin und jeder Psychiater auf die Patientenpersönlichkeiten nicht in der gleichen «genormten» und «durchschnittlichen» Weise, sondern - trotz anerkannter Fortbildungs-, Super- und Intervisionsrichtlinien - in der nur ihr bzw. ihm eigenen therapeutischen Haltung reagiert und dass sie bzw. er dabei aber trotzdem erfolgreich und wirtschaftlich behandeln kann? Ist ihnen bekannt, dass die «örtlichen Verhältnisse» weniger entscheidend für den Verlauf und Erfolg und damit auch für die Wirtschaftlichkeit - einer Behandlung sind als z.B. das Übertragungsund Gegenübertragungsgeschehen? Ist ihnen bekannt, dass die fachmedizinische PsychiatriePsychotherapie in den letzten Jahren einen zunehmenden Methodenspagat - ohne nachgewiesenen Wirksamkeitsverlust! - zwischen verschiedenen therapeutischen Ansätzen erlebt, bei welchem man annehmen kann, dass die Index- 
werte der Wirtschaftlichkeitskontrollen aller psychiatrisch-psychotherapeutischen Praxen immer weiter auseinanderdriften?

\section{Zweifel an der Wissenschaftlichkeit der Statistik}

Haben sich die Statistikerinnen und Statistiker der santésuisse einmal Gedanken darüber gemacht, dass bei psychiatrisch-psychotherapeutischen Behandlungen bewusste, unbewusste, individuelle, zwischenmenschliche und weitere komplexe Faktoren eine Rolle spielen und dass diese statistisch äusserst schwierig zu berechnen sind? Ist ihnen bekannt, dass die von der Statistik erfasste «Masse» der Psychiaterinnen und Psychiater mit grosser Wahrscheinlichkeit inhomogen ist, dass deshalb die psychiatrisch-psychotherapeutischen Praxen nicht über eine Leiste geschlagen werden dürften und dass die Daten dieser Fachrichtung viel differenzierter untersucht werden müssten? Kann deswegen die Rechnungsstellerstatistik der Krankenversicherer in bezug auf die spezifischen komplexen Verhältnisse der Psychiatrie-Psychotherapie nach seriösen, theoretisch fundierten Prinzipien als wissenschaftlich einwandfrei betrachtet werden?

\section{Unfaire Argumentation der santésuisse}

2 D'Souza C. Replik auf Leserbrief Dr. med. J. Bättig. Schweiz Ärztezeitung 2003;84(3):63-5.

3 D'Souza C. Warum «santésuisse auf dem Holzweg ist. Schweiz Ärztezeitung 2002;83(48):2607-8.

4 Marelli R. Die Psychiatrie und die Statistik. Schweiz Ärztezeitung 2002;83(48):2605-6

5 Schindler P. Von «Wirtschaftlichkeitskontrollen», der Abschaffung des Kontrahierungszwangs und dem drohenden Begräbnis unseres psychosozialen Gesundheitswesens. Schweiz Ärztezeitung 2002;83(48):2609-10.

6 Kurt H, Haemmerle P. Geld gespart - zu Lasten der psychisch Kranken. Schweiz Ärztezeitung 2002;83(48):2611

7 Wetter R. Abschaffung der ärztlichen Psychotherapie durch santésuisse? Schweiz Ärztezeitung 2002;83(51/52):2813.

8 Balmer R. Psychiatrie und Psychotherapie: Der Fallkostendurchschnitt ist untauglich für die Wirtschaftlichkeitskontrolle. Schweiz Ärztezeitung 2003; 84:(6):253-4.
Die Frage nach der «Tauglichkeit» müsste aber auch auf die Haltung der santésuisse und weiterer Kreise gegenüber der Psychiatrie-Psychotherapie erweitert werden. (Äusserungen über Leistungseinschränkungen lassen aufhorchen.)

In der oben zitierten Darstellung der santésuisse wird neben den Ausführungen über Wirtschaftlichkeitskontrollverfahren psychiatrischpsychotherapeutischer Praxen auch auf «krasse Beispiele» «rabenschwarzer» Schafe unter den Ärzten aufmerksam gemacht. Es ist aber geradezu polemisch, wenn die angeführten Beispiele nicht Psychiaterinnen und Psychiater, sondern unter anderem einen Allgemeinpraktiker mit einem «Umsatz von einer Million Franken» betreffen. Damit wird auf unfaire Weise suggeriert, dass die Psychiaterinnen und Psychiater ebenfalls rabenschwarze Schafe seien und ähnlich unverschämt hohe Umsätze generieren würden. Führte man das zitierte «krasse Beispiel» ad absurdum, dann müssten die Psychiaterinnen und Psychiater, um auf einen Umsatz von einer Million Franken zu kommen, bei einem Stundenansatz von Fr. 160 täglich (365 Tage im Jahr!) über 17 Stunden arbeiten!

\section{Vom Elfenbeinturm in die Öffentlichkeit}

Gegen Unverständnis und mangelnde Fairness uns Psychiaterinnen und Psychiatern sowie dadurch aber auch den Patientinnen und Patienten gegenüber muss man sich nun mit aller Bestimmtheit zur Wehr setzen [2]. Die Diskussion wurde öffentlich, nachdem sich einige Kolleginnen und Kollegen mit der Fragwürdigkeit der Rechnungsstellerstatistik der Krankenversicherer in Artikeln in der SÄZ auseinandergesetzt haben [3-8]. Es ist wichtig und dringend nötig, dass die Psychiaterinnen und Psychiater der ganzen Schweiz solidarisch Widerstand leisten. Man sollte sich aber nicht erst dann wehren, wenn einem die santésuisse droht. Der Schreibende ist 2001 laut Statistik bei einem Umsatz (Umsatz wohlverstanden!) von Fr. 183345 inklusive «veranlasste Kosten» (d.h. Rezepte usw.; kein Verdienst durch Medikamentenabgabe!) und einem Gesamtindex von 131 nochmals knapp davongekommen. Wie lange noch?

Doch nicht nur die einzelnen Betroffenen, sondern vor allem die psychiatrischen Fachgesellschaften sollten sich dringend der Frage annehmen, ob die Rechnungsstellerstatistik der Krankenversicherer über die Kosten psychiatrisch-psychotherapeutischer Behandlungen unter diesen Umständen und im Sinne der oben beschriebenen und für unser Fach spezifischen Komplexität wissenschaftlich hieb- und stichfest ist. Auch wäre es wünschenswert und könnte die Lage entspannen, wenn die Psychiaterinnen und Psychiater bzw. die psychiatrischen Fachgesellschaften die Statistikerinnen und Statistiker der santésuisse einladen und sie über solche Fragen und weitere - auch für eine wirtschaftliche Praxisführung - «als bedeutsam taxierte Faktoren» informieren könnten.

\section{Index- oder Behandlungspriorität?}

Schliesslich stellt sich auch die prinzipielle Frage nach den Prioritäten. Um was geht es eigentlich? Was ist wichtiger? Dass sich die Psychiaterinnen und Psychiater für die Statistik als «tauglich» erweisen, indem sie ihre Behandlungen zu einer indexabhängigen Durchschnittsmedizin zum Schaden ihrer Patientinnen und Patienten verkommen lassen? Oder sollte es nicht eher umgekehrt sein? Nämlich dass die Statistik für die spezifische Komplexität der Psychiatrie-Psychotherapie «tauglich» wird! 